\title{
Diseño y validación mediante Método Delphi de un cuestionario para conocer las características de la actividad física en personas mayores que viven en residencias Delphy Method validation and design of a questionnaire to assess physical activity characteristics in elderly from residences
}

Francisco José López Vidal, Africa Calvo Lluch

Universidad Pablo de Olavide (España)

Resumen. La promoción de la actividad física orientada a la salud y calidad de vida es una cuestión de gran interés en la sociedad. El objetivo del presente trabajo es el diseño y la validación de un cuestionario en español mediante un método de consulta a expertos, como es el Método Prospectivo de Delphi. El diseño del cuestionario fue orientado a conocer las características y la práctica de la actividad física en personas mayores que viven en centros residenciales. Se tuvo en cuenta para la validación a 13 expertos que agruparan conocimientos en actividad física, salud y tercera edad según sus perfiles. Los resultados que se obtuvieron muestran que el cuestionario es válido y fiable para conocer la existencia y características que presenta la actividad física en residencias para personas mayores. El cuestionario formado por 12 preguntas demuestra ser un instrumento válido para investigar hábitos y conductas físico-deportivas en la población de tercera edad que vive en residencias.

Palabras clave Cuestionario, Método Delphi, Actividad Física, Personas Mayores, Residencias.

Abstract. The promotion of health- and quality-of-life-oriented physical activity is a matter of great interest within the society. The objective of this study is to design and validate a questionnaire in Spanish language through a method based on experts' consultation, i.e. the Delphi Method. The design of the questionnaire was oriented to know the characteristics and practice of physical activity in elderly people living in residential centers. For its validation, the opinion of 13 experts with knowledge on physical activity, health, and seniors -based on their profile- was taken into account. The results obtained show that the questionnaire is valid and reliable to assess the presence and characteristics of physical activity in elderly residences. The questionnaire, comprising 12 items, proves to be a valid instrument to investigate habits and physical-sporting behavior in the elderly population living in residences.

Keywords Questionnaire, Delphi Method, Physical Activity, Elderly People, Residences.

\section{Introducción}

El cuestionario de actividad física para personas mayores en residencias es un instrumento diseñado para poder conocer de una manera clara y eficaz cómo es la actividad física de una persona mayor en su vida diaria en una residencia. Considerando el cuestionario como una herramienta de apoyo a los profesionales en el contexto de la actividad física, la calidad de vida y la promoción de la salud, de esta manera se identifica el grado de independencia y los estilos de vida vinculados a la actividad física de una población en particular, por medio de una serie de preguntas que presentan una formulación sencilla orientadas a unos sujetos de edad avanzada. La calidad de vida se trata de un término complejo que abarca la salud física y psicológica del sujeto, el grado de autonomía, las relaciones sociales, las creencias y valores personales y la relación con el entorno (Martínez \& Calvo, 2014). Para la preservación y desarrollo de la calidad de vida durante la vejez, la práctica de actividad física constituye un aspecto esencial para mantener la salud en las personas mayores (Pinillos, 2016). El deterioro progresivo que implica el envejecimiento ha centrado el interés de estudios que analizan las actitudes que tiene la sociedad sobre las personas mayores y cómo éstas influyen en la imagen que los mayores tienen de sí mismos (García \& Froment, 2018). A medida que envejecemos nuestra corporalidad y motricidad se debilita, debido no sólo al envejecimiento biológico sino también a una disminución de la actividad motora (Saüch,

Fecha recepción: 15-01-19. Fecha de aceptación: 31-05-19 Francisco José López Vidal franlopezvidal0@gmail.com
Castañer \& Hileno, 2013). También depende de la labor social de los profesionales en el cuidado de la personas mayores su estado de salud emocional. La Autoestima también se ve reforzada por el apoyo social, de manera que mientras más extensa sea la red de apoyo social, mejor Autoestima mantiene la persona mayor (Fernández \& Manrique-Abril, 2010). Del mismo modo, factores como la disminución de actividades y responsabilidades, limitaciones físicas, decrecimiento de la autonomía, pérdida de familiares o personas significativas y una conciencia más clara de la cercanía de la muerte, repercuten en el desarrollo de la autoestima (Gallego, Aguilar, Cangas, Lorenzo, Franco \& Mañas, 2012)

La necesidad de diseñar y validar este cuestionario es una muestra de la realidad actual en la presencia de actividad física en las personas mayores. La práctica de actividad física por parte de personas mayores a partir de los 60 años debe convertirse en una obligación, estando ello respaldado principalmente por las administraciones (Martínez \& Calvo, 2014). Las personas mayores que practican actividad física y deporte son todavía escasas en comparación a otros grupos sociales debido a la existencia de circunstancias, obstáculos o barreras (Campos, Jiménez-Beatty, González, Martín \& Hierro, 2011). Tras la revisión de cuestionarios de actividad física para personas mayores en el estudio de Moreno, GuiraoGoris, Cabrero-García \& Muñoz-Mendoza (2009), donde ninguno de los cuestionarios analizados se adecua a la población de personas mayores en residencias españolas según nuestro criterio, donde requerimos preguntas claras y concisas para que las personas encuestadas puedan entender las cuestiones y su respuesta sea adecuada a la realidad. Algunos revisados como el IPAQ-E, es un cuestionario que mide la actividad física y procede de la adaptación a personas 
mayores de 65 años de la versión corta del cuestionario internacional de la actividad física (IPAQ) y no está validado en nuestro país (Rubio, Tomás \& Muro, 2017). El cuestionario PASE-Physical Activity for Elderly fue el que contenía más aspectos relacionados con nuestro cuestionario, varias de sus preguntas orientadas a actividades poco presentes en nuestra sociedad como la jardinería o retirada de nieve por parte de las personas mayores en las residencias, lo que nos llevó a rechazarlo.

Para su validación hemos seleccionado el Método Prospectivo de Delphi, en un estudio de López-Gomez (2018), tras la revisión del uso del Método Prospectivo de Delphi desde los años 50 hasta la actualidad, se pone de manifiesto su uso y aplicación en distintas áreas de conocimiento como herramienta de consenso entre diversas opiniones entre expertos y retroalimentación. El Método Prospectivo de Delphi como instrumento de validación de cuestionarios ha sido ampliamente utilizado en numerosos estudios y ámbitos de conocimiento (Hung, Altschuld \& Lee, 2007). Tanto por su flexibilidad como por facilitar la toma de decisiones consensuadas, el Método Prospectivo de Delphi ha sido y es quizás una de las herramientas más empleadas en el mundo en el marco de la prospectiva (Aponte, Cardozo \& Melo, 2012). Por las características de este método de validación encontramos la utilidad de poder contactar con diferentes expertos de una manera no presencial. Okoli \& Pawlowski (2004) manifiestan que la utilización de la técnica Delphi es deseable cuando es difícil o no es factible reunir a los expertos participantes y también de esta manera se evita la confrontación directa de los expertos.

\section{Material y método}

En el diseño y aplicación de un cuestionario se debe procurar un equilibrio entre sencillez y claridad, sin profundizar en lo superficial (Carrasco \& Calderero, 2000). Para validar el cuestionario desarrollado que hemos empleado en nuestra investigación, se utilizó inicialmente el criterio de expertos a través del pronóstico cualitativo del Método Prospectivo de Delphi. Loo (2002), por su parte, destaca algunas características de este método que deben tenerse en cuenta en su aplicación: 1) La muestra está constituida por expertos seleccionados cuidadosamente. 2) Es importante mantener el anonimato de los expertos participantes con el fin de evitar influencias entre ellos. 3) Existencia de un investigador que dirige y controla todo el proceso. 4) Se produce una interacción como consecuencia de las circulaciones de informaciones realizadas entre investigador principal y panel de expertos. 5) El resultado obtenido hace referencia a las propuestas y sugerencias realizadas por el grupo de expertos.

Este método nos permitió consultar a un conjunto de expertos en el tema, para validar nuestra propuesta sustentándola en sus conocimientos, investigaciones, experiencia, estudios bibliográficos, etc. Según Varela-Ruiz, Díaz-Bravo \& García-Durán (2012), lo que se persigue con esta herramienta es obtener el grado de consenso o acuerdo entre especialistas sobre el problema planteado, en lugar de dejar la decisión a un solo profesional. Este método nos ha permitido que los expertos tuvieran tiempo suficiente para utilizar su criterio en el tiempo destinado al análisis de nuestro cuestionario. Es fácil de explicar la difusión del Método Prospectivo de Delphi porque este consiste en una técnica de obtención de información, basada en la consulta a expertos de un área, con el fin de obtener la opinión de consenso más fiable del grupo consultado (Renguant-Álvarez \& Torrado-Fonseca, 2016). Vamos a desarrollar cada uno de los pasos que hemos seguido para la valoración del cuestionario por orden numérico atendiendo a las pautas de Delphi;

Paso 1. Se elaboró un listado de expertos conocedores del tema específico de nuestro trabajo.

Paso 2. Se preguntó acerca del conocimiento sobre el tema a tratar con cada uno de los expertos, ofreciendo un mensaje claro y explicativo del motivo de su colaboración.

Paso 3. Se analizaron las respuestas y se identificaron las áreas e ítems en que estaban de acuerdo y en las que diferían.

La división de la validación en estos pasos nos ayudará a generar una mayor precisión de resultados, en otras validaciones de cuestionarios se destaca que con diferentes niveles de validación se pretendió garantizar que el cuestionario registre efectivamente aquello que pretende medir, buscando que tanto el diseño como su validación tengan una fuerte rigurosidad científica(Ortega, Calderón, Palao \& Puigcerver, 2008). Para este estudio siguiendo el primer paso citado anteriormente, se ha seleccionado una muestra de 13 expertos (2 gerentes de centros residenciales, 2 investigadores en actividad física y salud que trabajan con población de personas mayores y 9 profesores universitarios de Grado en Ciencias de la Actividad Física y del Deporte y Máster en Actividad Física y Salud de 5 universidades diferentes, de los cuales 7 de ellos son doctores en ciencias de la actividad física y del deporte), de esta manera hemos considerado abordar un listado con la presencia de expertos en diversas áreas que nos trasladan a los elementos principales de nuestro objeto de estudio. El criterio de selección de los expertos se hizo a partir de conseguir una visión colectiva entre profesionales del ejercicio físico en su mayoría y profesionales de la gestión de centros residenciales para personas mayores, de esta manera llegar a un consenso en sus respuestas.

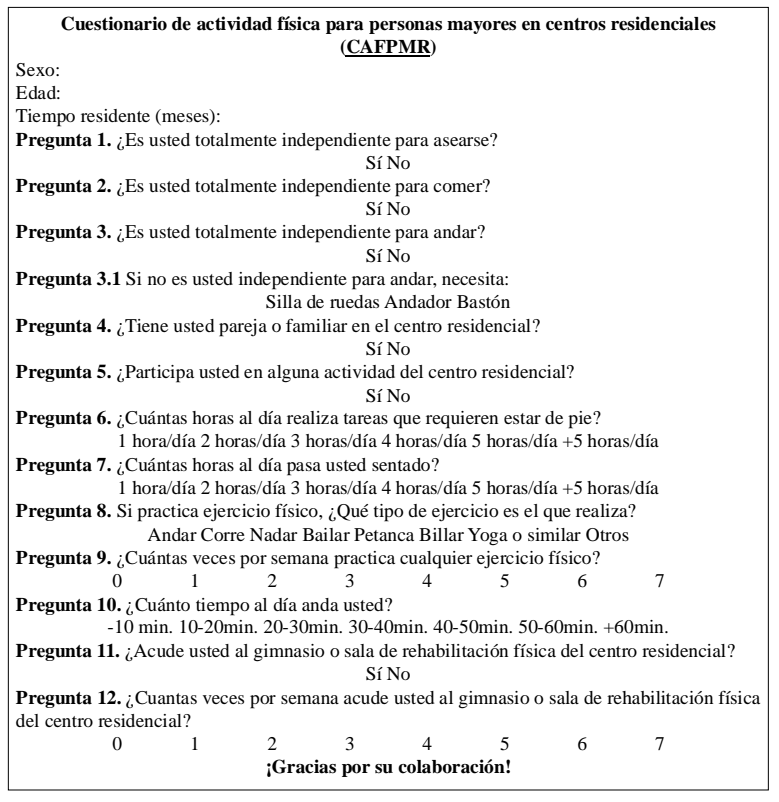


En el segundo paso nos dirigimos a los expertos seleccionados por medio de un contacto través del correo electrónico donde solicitamos su colaboración con la validación del cuestionario y su respuesta a las preguntas planteadas. Las respuestas a las preguntas nos aportaran una muestra de sus conocimientos en el objeto de estudio. Para ellos elaboramos preguntas acerca de la práctica de actividad física y el aporte de beneficios saludables, los conocimientos científicos y de existencia de programas de actividad física en centros residenciales. Además, se les proponía la oportunidad de realizar cualquier sugerencia a las preguntas (Almonacid-Fierro, Feu \& Vizuete, 2018), aportando cuestiones alternativas a las ya elaboradas o añadiendo cualquier otra que considerara oportuna. Las cuestiones que se lanzaron fueron; ¿qué opinión le merecen que la práctica de actividad física en las personas mayores pueda aportarles beneficios saludables y calidad de vida?, ¿conoce evidencias científicas que avalen el hecho de que la actividad física en personas mayores supone una mejora en su calidad de vida?, ¿sabe de la existencia de actividad física en centros residenciales para personas mayores públicos o privados?.

Tras las respuestas de los posibles expertos realizamos una valoración sobre el nivel de experiencia que tienen estas personas, de esta manera nos introducimos en el paso tercero, incluyendo en la evaluación aquellos que no contestaron. Evaluamos los niveles de conocimientos que poseen sobre Actividad Física y Centros Residenciales para personas mayores. Utilizamos una escala de evaluación creciente del uno al 10 para determinar el valor que se corresponde con el nivel de conocimiento o información que tienen sobre nuestro tema a estudiar y seleccionando como experto para la continuación de la validación del cuestionario aquellos que obtengan una calificación de ocho ó superior. Para dicha evaluación se obtuvo el siguiente cuadro:

\begin{tabular}{|c|c|c|c|c|}
\hline \multicolumn{5}{|c|}{ Evaluación de la respuesta de 1} \\
\hline Nombre & P1 & P2 & $\mathrm{P3}$ & \\
\hline Exp.1 & 10 & 9 & 7 & 8,66 \\
\hline Exp.2 & 6 & 5 & 4 & 5,00 \\
\hline Exp.3 & 10 & 10 & 8 & 9,33 \\
\hline Exp.4 & 8 & 10 & 8 & 8,66 \\
\hline Exp.5 & 10 & 10 & 9 & 9,66 \\
\hline Exp.6 & 9 & 10 & 9 & 9,33 \\
\hline Exp.7 & - & - & - & - \\
\hline Exp.8 & 9 & 8 & 10 & 9,00 \\
\hline Exp.9 & - & - & - & - \\
\hline Exp.10 & 9 & 8 & 9 & 8,66 \\
\hline Exp.11 & 10 & 10 & 9 & 9,66 \\
\hline Exp.12 & - & - & - & - \\
\hline Exp.13 & - & - & - & - \\
\hline
\end{tabular}

La tabla 1 muestra la valoración de las preguntas que se formularon a los expertos y la media aritmética que establece si son aptos o no para continuar como expertos en los siguientes pasos del protocolo de validación del cuestionario.

Contestaron a nuestro requerimiento nueve de los 13 expertos, y de las nueve respuestas, una de ellas obtuvo una calificación inferior a ocho.

De nuevo y por última vez contactamos con los expertos seleccionados para enviarles el cuestionario diseñado junto con un instrumento de observación donde cada experto podrá valorar su nivel de conocimiento sobre cada una de las preguntas marcando de uno a cuatro (siendo uno el valor de menor conocimiento y cuatro el de mayor) y pudiendo desarrollar observaciones junto a cada propuesta de pregunta.

A partir de aquí se calcula fácilmente el Coeficiente de
Conocimiento o Información (Kc), a través de la siguiente fórmula:

$$
\mathrm{Kc}=\mathrm{n}(0,1)
$$

Tabla 2 .

Coeficiente de conocimiento de los expertos

\begin{tabular}{cc}
\hline Expertos & Kc \\
\hline 1 & 0.8 \\
2 & 0.4 \\
3 & 0.9 \\
4 & 0.8 \\
5 & 0.9 \\
6 & 0.9 \\
7 & N.C. \\
8 & 0.9 \\
9 & N.C. \\
10 & 0.8 \\
11 & 0.9 \\
12 & N.C. \\
13 & N.C. \\
\hline
\end{tabular}

Siguiendo a George \& Mallery (1995) los valores cercanos al 0.8 se sitúan en el intervalo que los permite calificar como buenos y por lo tanto valores superiores como muy bueno. En el caso de los valores 0.5 o menos son denominados como débiles, por lo tanto los valores comprendidos entre $0,61-0,8$ lo consideramos con una fuerza moderada o buena.

Realizamos a continuación una pregunta que permitió valorar un grupo de aspectos que influyen sobre el nivel de argumentación o fundamentación del tema a estudiar. Las fuentes de argumentación que utilizamos son de una escala simple de Alto, Medio o Bajo.

Para ellos utilizamos la siguiente tabla:

\begin{tabular}{|c|c|c|c|}
\hline Fuentes de argumentación - Experto 1 & Alto & Medio & Bajo \\
\hline Análisis teóricos realizados por usted & $\mathrm{X}$ & & \\
\hline Su experiencia obtenida & $\mathrm{x}$ & & \\
\hline Trabajos de autores nacionales & $\mathrm{x}$ & & \\
\hline Trabajos de autores extranjeros & $\mathrm{x}$ & & \\
\hline Su conocimiento del estado del problema en el extranjero & $\mathrm{x}$ & & \\
\hline Su intuición & & $\mathrm{x}$ & \\
\hline Fuentes de argumentación - Experto 3 & Alto & Medio & Bajo \\
\hline Análisis teóricos realizados por usted & $\mathrm{X}$ & & \\
\hline Su experiencia obtenida & & $\mathrm{x}$ & \\
\hline Trabajos de autores nacionales & $\mathrm{x}$ & & \\
\hline Trabajos de autores extranjeros & $\mathrm{X}$ & & \\
\hline Su conocimiento del estado del problema en el extranjero & $\mathrm{x}$ & & \\
\hline Su intuición & $\mathrm{x}$ & & \\
\hline Fuentes de argumentación - Experto 4 & Alto & Medio & Bajo \\
\hline Análisis teóricos realizados por usted & & $\mathrm{X}$ & \\
\hline Su experiencia obtenida & $\mathrm{x}$ & & \\
\hline Trabajos de autores nacionales & $\mathrm{x}$ & & \\
\hline Trabajos de autores extranjeros & $\mathrm{x}$ & & \\
\hline Su conocimiento del estado del problema en el extranjero & & $\mathrm{x}$ & \\
\hline$\underline{\text { Su intuición }}$ & $\mathrm{X}$ & & \\
\hline Fuentes de argumentación - Experto 5 & Alto & Medio & Bajo \\
\hline Análisis teóricos realizados por usted & & $\mathrm{X}$ & \\
\hline Su experiencia obtenida & $\mathrm{x}$ & & \\
\hline Trabajos de autores nacionales & $\mathrm{x}$ & & \\
\hline Trabajos de autores extranjeros & $\mathrm{x}$ & & \\
\hline Su conocimiento del estado del problema en el extranjero & $\mathrm{x}$ & & \\
\hline$\underline{\text { Su intuición }}$ & $\mathrm{x}$ & & \\
\hline Fuentes de argumentación - Experto 6 & Alto & Medio & Bajo \\
\hline Análisis teóricos realizados por usted & $\mathrm{X}$ & & \\
\hline Su experiencia obtenida & & $\mathrm{x}$ & \\
\hline Trabajos de autores nacionales & $\mathrm{x}$ & & \\
\hline Trabajos de autores extranjeros & & $\mathrm{x}$ & \\
\hline Su conocimiento del estado del problema en el extranjero & $\mathrm{X}$ & & \\
\hline Su intuición & $\mathrm{x}$ & & \\
\hline Fuentes de argumentación - Experto 8 & Alto & Medio & Bajo \\
\hline Análisis teóricos realizados por usted & & $\mathrm{X}$ & \\
\hline Su experiencia obtenida & $\mathrm{x}$ & & \\
\hline Trabajos de autores nacionales & $\mathrm{x}$ & & \\
\hline Trabajos de autores extranjeros & & $\mathrm{x}$ & \\
\hline Su conocimiento del estado del problema en el extranjero & $\mathrm{X}$ & & \\
\hline Su intuición & $\mathrm{X}$ & & \\
\hline Fuentes de argumentación - Experto 10 & Alto & Medio & Bajo \\
\hline Análisis teóricos realizados por usted & $\mathrm{X}$ & & \\
\hline Su experiencia obtenida & $\mathrm{x}$ & & \\
\hline Trabajos de autores nacionales & $\mathrm{x}$ & & \\
\hline Trabajos de autores extranjeros & $\mathrm{x}$ & & \\
\hline Su conocimiento del estado del problema en el extranjero & $\mathrm{x}$ & & \\
\hline Su intuición & $\mathrm{x}$ & & \\
\hline Fuentes de argumentación - Experto 11 & Alto & Medio & Bajo \\
\hline Análisis teóricos realizados por usted & $\mathrm{X}$ & & \\
\hline Su experiencia obtenida & & $\mathrm{x}$ & \\
\hline Trabajos de autores nacionales & $\mathrm{X}$ & & \\
\hline Trabajos de autores extranjeros & $\mathrm{X}$ & & \\
\hline Su conocimiento del estado del problema en el extranjero & $\mathrm{x}$ & & \\
\hline Su intuición & $\mathrm{x}$ & & \\
\hline
\end{tabular}


Como se puede observar no existen ni el experto dos, siete, nueve, 12 ni 13 ya que no contestaron a ninguno de los requerimientos por parte del investigador.

Posteriormente se determinaron los aspectos de mayor influencia, los aspectos que influyen sobre el nivel de argumentación o fundamentación del tema a estudiar permitieron calcular el Coeficiente de Argumentación (Ka) de cada experto.

\begin{tabular}{lccc} 
Tabla 4. & & & \\
Valores de mayor influencia & Alto & Medio & Bajo \\
\hline Fuentes de argumentación & 0.3 & 0.2 & 0.1 \\
\hline Análisis teóricos realizados por usted & 0.5 & 0.4 & 0.2 \\
Su experiencia obtenida & 0.05 & 0.05 & 0.05 \\
Trabajos de autores nacionales & 0.05 & 0.05 & 0.05 \\
Trabajos de autores extranjeros & 0.05 & 0.05 & 0.05 \\
Su conocimiento del estado del problema en el extranjero & 0.05 & 0.05 & 0.05 \\
Su intuición
\end{tabular}

Una vez obtenido los valores del Coeficiente de Conocimiento (Kc) y el Coeficiente de Argumentación (Ka) se procedió a obtener el valor del Coeficiente de Competencia (K) que finalmente es el coeficiente que determinó en realidad que expertos se tomaba en consideración para trabajar en esta investigación. Este coeficiente $(\mathrm{K})$ se calculó de la siguiente forma: $\mathrm{K}=0,5(\mathrm{Kc}+\mathrm{Ka}$ ), donde: $\mathrm{K}$ : Coeficiente de Competencia, Kc: Coeficiente de Conocimiento y Ka: Coeficiente de Argumentación

Posteriormente se establecieron como criterios de selección los siguientes:

- Coeficiente de Competencia Alta: 0,8 $<\mathrm{K}<1,0$

- Coeficiente de Competencia Media: 0,5 $<\mathrm{K}<0,8$

- Coeficiente de Competencia Baja: $\mathrm{K}<0,5$

Para la finalización del diseño del cuestionario, realizamos cambios puntuales de tipo cualitativo en la formulación de preguntas y la modificación de algunos aspectos detallados en las posibles respuestas siempre atendiendo a lo establecido por los expertos en el punto anterior.

Como hemos comentado al inicio de este apartado, las observaciones de los expertos seleccionados se tuvieron en cuenta para hacer cambios en la formulación de las preguntas del cuestionario, nos sirvió como tormenta de ideas todas las aportaciones que ofrecieron los ocho expertos finales. Gracias a la claridad y concreción de las observaciones no fue necesario hacer una segunda vuelta.

Todas las preguntas que completan el cuestionario han sido revisadas detalladamente para adecuarlas a la población de destino bajo la supervisión de todos los expertos y adaptando las cuestiones desde los marcos referenciales de cuestionarios como el IPAQ, que fue desarrollado para medir la actividad física en personas mayores, sin embargo, algunos autores indican que este cuestionario ha demostrado no ser idóneo para medir la AF en personas mayores de 50 años (Grimm, Swartz, Hart, Miller \& Strath, 2012). Las encuestas poblacionales hacen patente su vulnerabilidad, en este subgrupo poblacional considerado físicamente inactivo (López, Santos, Navarro, Arévalo, García \& Latorre, 2016).

\section{Resultados}

Con respecto a la validez de las preguntas iniciales que se habían establecido, tras la validación por parte del juicio de los expertos, las modificaciones que se realizaron fueron las siguientes:
- Eliminación de una de las preguntas.

- Modificación en la estructura de cuatro preguntas.

- Perfeccionamiento en cuanto a la redacción de preguntas y selección de respuesta para facilitar la comprensión y generar fluidez en la respuesta.

Según Burgos (2006), son muy pocos los cuestionarios que se diseñan y validan siguiendo procesos metodológicos adecuados. Por lo que hemos observado que esta validación nos acerca a la realidad de conocer los resultados con el carácter más fiable posible. Tras la valoración final de los expertos y las recomendaciones de modificación en alguno de los apartados iniciales del cuestionario, establecemos el resultado final que corresponde al cuestionario en sí.

Inicialmente se establecen tres datos sociodemográficos a rellenar por el encuestado, sexo, edad y tiempo residente en meses, estas variables nos ayudarán a establecer grupos diferenciados en el estudio estadístico y correlaciones con aporte de interés. Por ello, es necesario identificar aquellos factores sociodemográficos, clínicos y comportamentales que se encuentren ligados a la práctica de la actividad física, con el propósito de implementar programas de intervención que ayuden a las personas mayores a adoptar un comportamiento físicamente más activo (Menezes, Dos-Santos-Silva, Tribess, Romo-Perez \& Virtuoso-Júnior, 2015). Las preguntas uno, dos y tres corresponden a actividades básicas de la vida diaria como son el aseo, la alimentación y andar, de esta manera podremos conocer la independencia de los residentes frente a estas actividades. Si el sujeto no es independiente para andar, podremos saber el implemento que utilizar para desplazarse, así podemos profundizar aún más en su autosuficiencia. Las preguntas cuatro y cinco se orientan a la actividad social de los encuestados, la cuestión cuatro formula si la persona mayor tiene pareja o familiar en el centro residencial y la cinco si participa en las tareas y actividades del propio centro, actividades que propician el trabajo en grupo, el coloquio, la interacción entre los residentes y el propio movimiento especialmente haciendo referencia a la motricidad fina en trabajos de manualidad principalmente.

La inactividad física o sedentarismo tiene una primera medida en las horas que pasa una persona sentada y de pie, ambas preguntas se encuentran en las preguntas seis y siete conociendo la proporción de tiempo en horas que pasan los sujetos encuestados tanto sentados como de pie. Esta presencia del sedentarismo es lo que está llevando a los profesionales procedentes del ámbito sanitario a adoptar medidas encauzadas a la promoción de la actividad física de la sociedad en general y las personas mayores en particular (Salinas, Cocca, Mohamed \& Viciana, 2010). En las preguntas ocho y nueve vamos a conocer el ejercicio físico que práctica, en el caso de que se practique y la frecuencia semanal con la que se ejerce la propia práctica. González, Martín, Jiménez-Beatty, Campos \& Del Hierro (2010) utilizaron como técnica de investigación la entrevista estructurada, siendo la población objeto de estudio personas mayores, se les preguntó el modo de realización de la práctica física dentro de dos opciones de respuesta: «con presencia de profesor o sin presencia de profesor»; en otra de las cuestiones demandadas se les interrogó sobre el nivel de organización de la actividad física realizada, haciendo referencia a la práctica físico deportiva organizada o dirigida o por el contrario auto organizada, es 
decir de carácter libre. A continuación en la 10 conocemos el tiempo estimado que anda diariamente cada persona mayor como actividad física más usual entre la población envejecida. Las dos últimas cuestiones, 11 y 12 hacen referencia a si acuden o no los residentes al gimnasio o sala de rehabilitación física de la residencia y a su frecuencia semanal de asistencia.

El cuestionario desarrollado ha sido aplicado mediante una prueba piloto a 424 personas. Para comprobar que los datos siguen una distribución normal hemos aplicado el test de Kolmogorov-Smirnov (conocido como test Lilliefors, suponiendo media y varianza desconocida) y el test de Shapiro Wilk. La hipótesis planteada es la siguiente para ambos test. Antes de proceder a la recogida de datos, se pidió permiso a los responsables de las diferentes organizaciones que participaron en el estudio.

\begin{tabular}{|c|c|c|c|c|c|c|}
\hline \multicolumn{7}{|c|}{ Kolmogorov-Smirnov ${ }^{a}$} \\
\hline & Estadístico & $\mathrm{gl}$ & Sig. & Estadístico & $\mathrm{gl}$ & Sig. \\
\hline Sexo &, 405 & 424 & 000 & 613 & 424 &, 000 \\
\hline InterEdad & ,274 & 424 & 000 & ,856 & 424 & ,000 \\
\hline TiempoResid & ,209 & 424 & ,000 & ,814 & 424 & 000 \\
\hline P1 & ,370 & 424 & ,000 & ,632 & 424 & 000 \\
\hline P2 & 537 & 424 & 000 & ,290 & 424 & ,000 \\
\hline P3 & ,435 & 424 & ,000 & ,585 & 424 & ,000 \\
\hline P3.1 & ,200 & 424 & 000 & 856 & 424 & ,000 \\
\hline P4 & ,508 & 424 & 000 & ,441 & 424 & , 000 \\
\hline P5 & ,441 & 424 & ,000 & ,578 & 424 & , 000 \\
\hline P6 & ,238 & 424 & ,000 & 869 & 424 & ,000 \\
\hline P7 & 360 & 424 & ,000 & 696 & 424 & ,000 \\
\hline P8 & ,420 & 424 & 000 & ,650 & 424 & ,000 \\
\hline P9 & 167 & 424 & 000 & 879 & 424 & 000 \\
\hline P10 & 156 & 424 & 000 & 881 & 424 & 000 \\
\hline P11 & 433 & 424 & 000 & ,588 & 424 & , 000 \\
\hline P12 & ,185 & 424 & 000 & 881 & 424 & ,000 \\
\hline
\end{tabular}

Como se observa en la tabla según las hipótesis planteadas en ambos test, para cada una de las variables el nivel de significación es menor de 0,05. Lo que nos permite aceptar la hipótesis nula, es decir, se puede afirmar que los datos proceden de una distribución normal.

\section{Conclusiones}

El objetivo de este trabajo ha sido diseñar y validar un cuestionario para su aplicación en centros residenciales para personas mayores en España. A partir de los resultados que hemos obtenido, se puede concluir que el instrumento validado dispone de los dispositivos óptimos de validez y fiabilidad para dar respuesta al objetivo planteado, nos permite analizar las características de la actividad física en la población de personas mayores que vive en centros residenciales, concretamente aquellos que no presentan un deterioro cognitivo y puede responder a las preguntas formuladas en el cuestionario.

Con el diseño y la validación de este cuestionario, los investigadores y responsables de los servicios de actividad física y deportes de las residencias para personas mayores cuentan con un instrumento muy útil, práctico y sencillo para conocer y poder registrar las características de la actividad física en la población de residentes.

Esta herramienta está enfocada a la actividad física y su relación con la salud y la calidad de vida, debido a su carácter de obtener información acerca de los hábitos diarios que implican actividad física, así como la práctica de ejercicios concretos en una población que se asocia a la dependencia.
En lo relativo a la capacitación de los expertos y a su dedicación en los pasos de la validación del instrumento destacamos el interés que han mostrado aquellos que accedieron a seguir el protocolo, un punto fuerte, el haber contado para la validación del cuestionario con un grupo multidisciplinar de expertos, con una experiencia consolidada en el ámbito del ejercicio físico y el envejecimiento. En definitiva la elaboración de este cuestionario ayuda a fomentar programas preventivos en la tercera edad para tratar de lograr un envejecimiento saludable.

\section{Referencias}

Almonacid-Fierro, A., Feu, S., \& Vizuete, M. (2018). Validación de un cuestionario para medir el Conocimiento Didáctico del Contenido en el profesorado de Educación Física. Retos. Nuevas tendencias en Educación Física, Deporte y Recreación, 34, 132-137.

Aponte, G., Cardozo, M.A., \& Melo, R.M. (2012). Método DELPHI: aplicaciones y posibilidades en la gestión prospectiva de la investigación y desarrollo. Revista Venezolana de Análisis de Coyuntura, vol. XVIII, núm. 1, enero-julio, 2012, pp. 41-52.

Burgos, R. (2006). Metodología de investigación y escritura científica en clínica. Escuela Andaluza de Salud Pública.

Campos, A., Jiménez-Beatty, J. E., González, M. D., Martín, M., \& Hierro, D. (2011). Demanda y percepción del monitor de las personas mayores en la actividad física y deporte en España. Revista de Psicología del Deporte, 20(1), 61-77.

Carrasco, J. B., \& Calderero, J. F. (2000). Aprendo a investigar en Educación. Madrid: RIALP.

Fernández, R., \& Manrique-Abril, F.G. (2010). Rol de la enfermería en el apoyo social del adulto mayor. Enfermería Global, 19, 1-9.

Gallego, J., Aguilar, J. M., Cangas, A. J., Lorenzo, J. J., Franco, C., \& Mañas, I. (2012). Programa de Natación Adaptada para Personas Mayores Dependientes: Beneficios Psicológicos, Físicos y Fisiológicos. Revista de Psicología del Deporte, 21(1), 125-133.

García, A.J., \& Froment, F. (2018). Beneficios de la actividad física sobre la autoestima y la calidad de vida de personas mayores. Retos. Nuevas tendencias en Educación Física, Deporte y Recreación, 33, 3-9.

George, D., \& Mallery, P. (1995). SSPS/PC + Step by: A Simple Guide and reference. Belmont: Wadsworth Publishing Company.

González, M. D., Martín, M., Jiménez-Beatty, J. E., Campos, A., \& Del Hierro D. (2010). Hábitos de actividad física de las personas mayores en España y condición social. Apunts. Educación Física y Deportes, 101, 87-98.

Grimm EK, Swartz AM, Hart T, Miller NE, Strath SJ. Comparison of the IPAQ-Short form and accelerometry predictions of physical activity in older adults. J Aging Phys Act. 2012;20:64-79.

Hung H.L, Altschuld, J.W., \& Lee, Y.F. (2008): Methodological and conceptual issues confronting a cross-country Delphi study of educational program evaluation. Evaluation and Program Plannin, 31, 191-198. 
Loo, R. (2002). The Delphi method: A powerful tool for strategic management. Policing: An International Journal of Police Strategies and Management, 25(4), 762-769.

López, R. C., Santos, M. A., Navarro, A. V., Arévalo, J. M., García, F., \& Latorre, P. A. (2016). Determinantes sociodemográficos y nivel de actividad física en la población de la provincial de Jaén mayor de 18 años. Retos. Nuevas Tendencias en Educación Física, Deportes y Recreación, 29, 13-16.

López-Gómez, E. (2018). El método Delphi en la investigación actual en educación: una revisión teórica y metodológica. Educación XX1, 21(1), 17-40.

Martínez, J., \& Calvo, Á. (2014). Calidad de vida percibida y su relación con la práctica de actividad física en el ámbito laboral. Un estudio piloto. Retos. Nuevas Tendencias en Educación Física, Deportes y Recreación, 25, 53-57.

Menezes, A. S., dos-Santos-Silva, R. J., Tribess, S., RomoPérez, V., \& Virtuoso-Júnior, J. S. (2015). Inactividad física $\mathrm{y}$ factores asociados en personas mayores en Brasil. Revista Internacional de Medicina y Ciencias de la Actividad Física y el Deporte, 15(60), 773-784.

Moreno, J., Guirao-Goris, J.A., Cabrero-García, J., \& MuñozMendoza, C.L. (2009). Revisión estructurada de los cuestionarios y escalas que miden la actividad física en los adultos mayores y ancianos, 23(4).

Okoli, C., \& Pawlowski, S.D. (2004). The Delphi method as a research tool: An example, design considerations and applications. Information \& Management, 42, 15-29.

Ortega, E., Calderón, A., Palao, J. M., \& Puigcerver, M. C.
(2008). Diseño y validación de un cuestionario para evaluar la actitud percibida del profesor en clase y de un cuestionario para evaluar los contenidos actitudinales de los alumnos durante las clases de educación física en secundaria. Retos. Nuevas tendencias en Educación Física, Deporte y Recreación, 14, 22-29.

Pinillos, M. (2016). Efectos positivos del entrenamiento de karate en las capacidades cognitivas asociadas a la edad. Revista Internacional de Medicina y Ciencias de la Actividad Física y el Deporte, 16(63), 537-559.

Reguant-Álvarez, M. \& Torrado-Fonseca, M. (2016). El método Delphi. REIRE, Revista d'Innovació i Recerca en Educació, 9(1), 87-102.

Rubio Castañeda, F., \& Tomás Aznar, C., \& Muro Baquero, C. (2017). Medición de la actividad física en personas mayores de 65 años mediante el IPAQ-E: validez de contenido, fiabilidad y factores asociados. Revista Española de Salud Pública, 91, 1-12.

Salinas, F., Cocca, A., Mohamed, K., \& Viciana, J. (2010). Actividad física y sedentarismo: Repercusiones sobre la salud y calidad de vida de las personas mayores. Retos. Nuevas tendencias en Educación Física, Deporte y Recreación, 17, 126-129.

Saüch, G, Castañer, M., \& Hileno, R. (2013). Valorar la capacidad de equilibrio en la tercera edad. Retos. Nuevas tendencias en Educación Física, Deporte y Recreación, 23, 48-50.

Varela-Ruiz, M., Díaz-Bravo, L., \& García-Durán, R. (2012). Descripción y usos del método Delphi en investigaciones del área de la salud. Revista Investigación en Educación Médica, 1(2), 90-95.

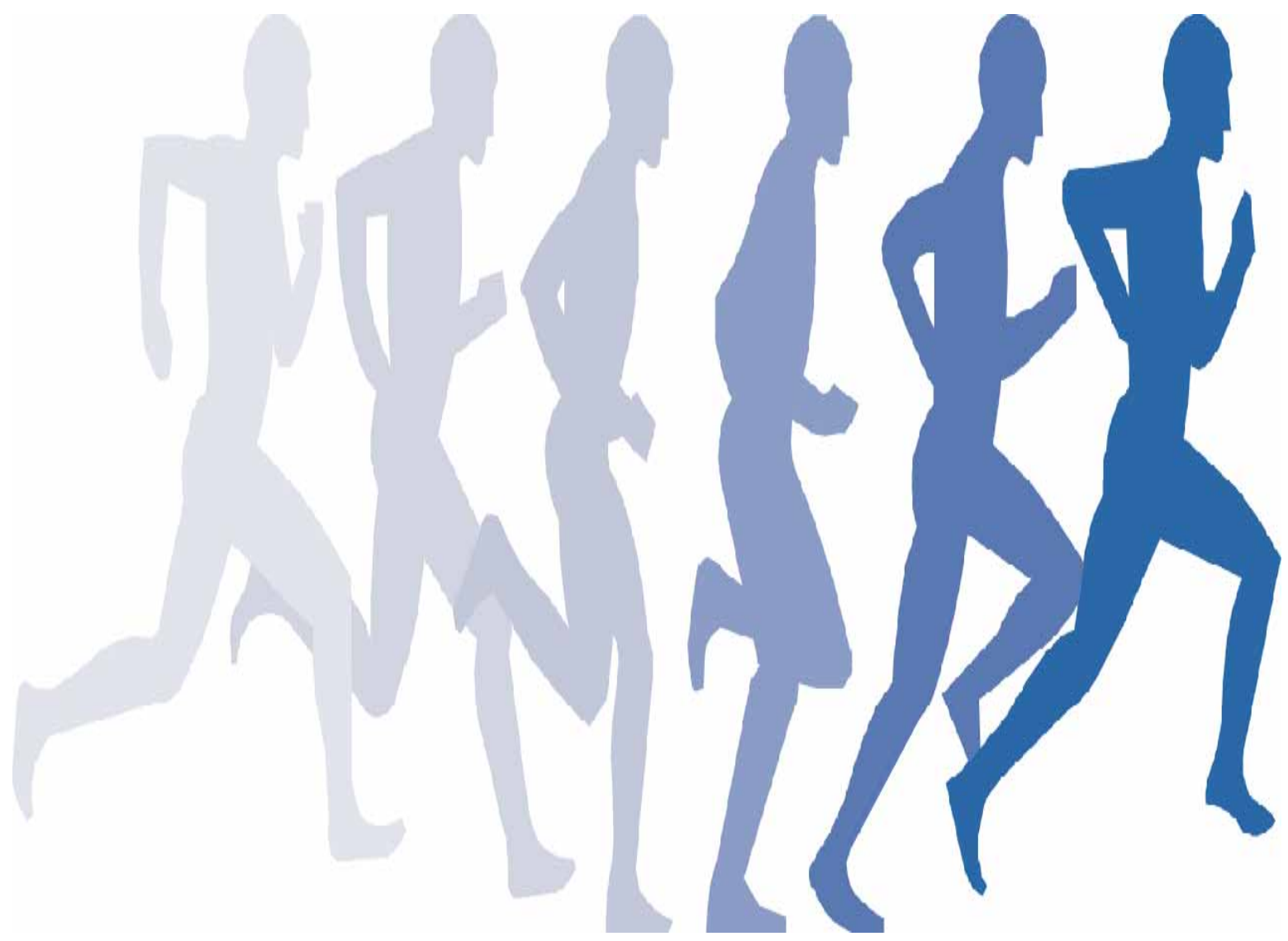

